\title{
TNFSFR1A R92Q mutation, autoinflammatory symptoms and multiple sclerosis in a cohort from Argentina
}

\author{
Marcelo A. Kauffman • Dolores Gonzalez-Morón • \\ Orlando Garcea $\cdot$ Andrés María Villa
}

Received: 23 December 2010/ Accepted: 23 April 2011

(C) Springer Science+Business Media B.V. 2011

\begin{abstract}
Systemic autoinflammatory diseases are genetic disorders characterized by seemingly unprovoked inflammation, without major involvement of the adaptive immune system. Among them it is recognized the TNF receptor associated periodic syndrome (TRAPS) caused by mutations in the TNFRSF1A gene and characterized by symptoms such as recurrent high fevers, rash, abdominal pain, arthralgia and myalgia. Recent studies have recognized the potential role of TNFRSF 1A mutations in Multiple Sclerosis (MS). Our aim was to investigate the role of TNFRSF1A R92Q gene mutation in a cohort of 90 Argentinean MS patients, where we determined the frequency of the TNFRSF1A R92Q mutation. We also compared autoinflammatory symptoms, MS clinical characteristics and treatment response and tolerability in R92Q carriers and non-carriers. Also, we used a case-control study design to obtain the genotypes of 78 healthy controls and assess the role of this mutation as a risk factor for MS. We found that five patients (5.5\%) carried the R92Q mutation, four reported autoinflammatory symptoms previous to MS onset. We found no differences in MS clinical features, treatment response and tolerability between carriers and non-carriers. R92Q mutation was more frequent
\end{abstract}

M. A. Kauffman $(\bowtie) \cdot$ D. Gonzalez-Morón

Neurogenetics Clinic, Neurology Division, Hospital JM Ramos

Mejia, School of Medicine, University of Buenos Aires,

Neuroimmunology Unit, 609-1221 Urquiza, Buenos Aires,

Argentina

e-mail: marcelokauffman@marcelokauffman.info

M. A. Kauffman

Neurogenetics Lab, Neurology Division, Sanatorio V Franchin, Buenos Aires, Argentina

O. Garcea · A. M. Villa

Neuroimmunology Unit, Neurology Division, Hospital JM

Ramos Mejia, Buenos Aires, Argentina in MS patients as compared to controls. This increases the risk to develop MS in about 4.5 times. The TNFRSF1A R92Q mutation is a common finding in Argentinean MS patients. This genetic variant might be a risk factor for MS.

Keywords Multiple sclerosis - Tumour necrosis factor receptor - Genetics · Risk factor

\section{Introduction}

Tumor necrosis factor receptor (TNRF) 1-associated periodic syndrome (TRAPS; Mendelian Inheritance in Man [MIM] 142680) is an autoinflammatory inherited disorder characterized by recurrent bouts of fever, abdominal pain, myalgia, rash, arthralgia, conjunctivitis and rhinopharingeal involvement that typically last for several days up to weeks [1]. It was initially termed as familial Hibernian fever after the description of an Irish-Scotish family in 1982 [2]. It is caused by mutations in the tumor necrosis factor (TNF) receptor superfamily 1A (TNFRSF1A; MIM 191190) gene, which encodes the TNF $\alpha 55$-kd receptor. More than 50 different TNFRSF1A mutations have been described elsewhere [3]. However, the R92Q substitution is the most frequent and widespread mutation identified in patients suffering from TRAPS. Carriers of this genetic defect present milder and more heterogeneous symptoms [4]. Multiple Sclerosis is an autoimmune, recurrent and chronic disorder of the central nervous system (CNS) that involves different genetic factors in its complex etiology. Recent reports have indicated the potential role of the TNFRSF1A gene among these genetic factors [5].

Carriers of the TNFRSF1A R92Q mutation presenting the apparent coexistence of typical MS and late-onset TRAPS have been identified [6]. On the other hand, a few cases of TRAPS with CNS involvement with response to 
anti-TNF therapy (so called CNS-TRAPS) have also been described [7]. Therefore, it is unclear whether these patients present the coexistence of two different and independent disorders or if they are affected by a unique autoinflammatory disorder with CNS involvement mimicking MS [8]. Because anti-TNF therapeutics have proven deleterious in MS [9], this controversy exceeds etiological grounds and raises the question about their role, if any, in a subset of MS (-like) disorders.

The main objectives of this study were to assess the frequency of TNFRSF 1A R92Q mutation in a cohort of MS Argentinean patients and to investigate the role of this mutation in MS clinical characteristics. Furthermore, we explored the role of this mutation as a genetic risk factor for developing MS.

\section{Methods}

\section{Study population}

We studied a cohort of 90 Argentinean patients with relapsing remitting MS who were consecutively recruited at the Ramos Mejia Hospital MS clinic in the city of Buenos Aires from March 2005 to January 2006. The study was reviewed and approved by the local Research Ethics Committee. It have therefore been performed in accordance with the ethical standards laid down in the 1964 Declaration of Helsinki. We obtained written informed consent from each patient before blood sampling. All patients underwent a standard battery of examinations, including medical history, physical and neurological examinations, screening laboratory tests and brain magnetic resonance imaging. Diagnosis was made in accordance with the McDonald criteria [10].

A detailed history including MS onset and course, treatment history, response and tolerability and family history were taken from each patient at recruitment. To assess clinical status we used the Expanded Disability Status Scale (EDSS) and then calculated the progression index (PI $=$ EDSS/disease duration in years). A personal and family history of autoinflammatory symptoms was retrospectively investigated in carriers of the R92Q TNFRSF1A mutation.

In order to explore the role of the R92Q mutation as a genetic risk factor for MS, we included 84 unrelated healthy controls who were matched for ethnic background, gender and age, without positive history of neurological, autoimmune or psychiatric disease.

\section{TNFSF1A R92Q genotyping}

To isolate genomic DNA from whole blood we used a Flexigene kit, as described by the manufacturer (Qiagen,
Hilden, Germany). We assessed the presence of the TNFSF1A R92Q mutation blind to clinical status by polymerase chain reaction (PCR)-restriction fragment length polymorphism assay using primers: $5^{\prime} G G G A C A C$ TGCATGGATGTGAG and $5^{\prime}$ GAGGAAGTGACGAGGGA $C A$. Annealing conditions were at $60^{\circ} \mathrm{C}$ for $45 \mathrm{~s}$ and 35 cycles. A $10 \mu 1$ sample of PCR product was digested with $4 \mathrm{U}$ of MspI in a final volume of $20 \mu \mathrm{l}$. Mutant alleles yielded two fragments of 191 and $237 \mathrm{bp}$ and wild type alleles yielded three fragments of 36, 155 and 237 bp. Fragments were resolved by $2.5 \%$ agarose gel electrophoresis.

\section{Statistical analysis}

Hardy-Weinberg equilibrium was tested by exact test. MS clinical and therapeutic features were compared between R92Q carriers and non-carriers fitting different regression models. A dominant heredity model was assumed to test the role of R92Q mutation as a genetic susceptibility factor to develop MS. We used the chi-square test to compare allelic frequencies between patients and controls. Significance level was set at 0.05 . All statistical analyses were performed using Stata version 9 for Windows.

\section{Results}

Five patients tested positive for the TNFRSF1A R92Q mutation which amounts to a $5.5 \%$ prevalence in our population. A summary of their characteristics is shown in Table 1. All of them had a definite diagnosis of MS according to the McDonald criteria and were born in Argentina. Four of them were women. The mean age of our cohort was 44.4 years, whereas the mean age at disease onset was 31 years (range 21-42 years). They were followed for a mean of 13.4 years (range 2-19 years) at the time of blood collection. All of them showed a typical course of MS without evidence of a more severe or progressive disease. We also obtained typical MS findings on CSF and MRI. Therapeutic response and tolerability were consistent with what is typically expected in these patients.

R92Q carriers differed from non-carriers in the MS first relapse location. We found more histories of first relapse locations in brain stem or cerebellum in R92Q carriers than in non-carriers $(P=0.03$; OR $=7.5)$. However, we found no differences regarding other MS features.

Four of the five patients that were positive for R92Q mutation had a medical history of autoinflammatory symptoms. These mainly included arthralgias, headache, migratory erythematous rash, fever and recurrent pharyngitis that required surgical tonsil excision. These symptoms were present before the clinical onset of MS. We found no 
Table 1 Characteristics of patients positive for R92Q mutation

\begin{tabular}{|c|c|c|c|c|c|c|c|c|c|}
\hline Patient & Age & Sex & $\begin{array}{l}\text { Age at } \\
\text { MS Onset }\end{array}$ & $\begin{array}{l}\text { Initial } \\
\text { manifestation } \\
\text { of MS }\end{array}$ & EDSS & $\begin{array}{l}\text { EDSS/time } \\
\text { index }\end{array}$ & $\begin{array}{l}\text { CSF } \\
\text { OCB }\end{array}$ & $\begin{array}{l}\text { Autoinflammatory } \\
\text { Symptoms }\end{array}$ & Other diseases \\
\hline $1(\mathrm{VG})$ & 49 & $\mathrm{~F}$ & 35 & Hemiparesis right & 6.5 & 0.5 & Present & Pharyngitis/recurrent fever ${ }^{\mathrm{a}}$ & None \\
\hline $2(\mathrm{CU})$ & 47 & M & 28 & Optic Neuritis & 6 & 0.3 & NI & $\begin{array}{l}\text { Recurrent and severe } \\
\text { Pharyngitis }\end{array}$ & $\begin{array}{l}\text { Psoriasis/Seizures } \\
\text { in the infancy }\end{array}$ \\
\hline $3(\mathrm{OM})$ & 43 & $\mathrm{~F}$ & 29 & Vertigo/diplopia & 2.5 & 0.2 & Present & Pharyngitis/recurrent fever ${ }^{\mathrm{a}}$ & None \\
\hline $4(\mathrm{ML})$ & 39 & $\mathrm{~F}$ & 21 & $\begin{array}{l}\text { Diplopia/Facial } \\
\text { nerve palsy }\end{array}$ & 2 & 0.1 & Present & Artrhalgias/Rash & None \\
\hline $5(\mathrm{NO})$ & 44 & $\mathrm{~F}$ & 42 & Diplopia & 3 & 1.5 & NI & None & None \\
\hline
\end{tabular}

$M S$ Multiple Sclerosis, EDSS expanded disability scale score, $C S F$ cerebrospinal fluid, $O C B$ oligoclonal bands

${ }^{a}$ Febrile bouts typically referred lasting 5 or 6 days

patients with signs of TRAPS-related renal disease such as proteinuria, renal failure, elevated creatinine or urea levels. We excluded other autoimmune diseases, with the exception of psoriasis in one patient. Furthermore, first-degree relatives referred history of symptoms suggestive of an autoinflammatory disorder. In one family, four of the six first-degree relatives interviewed had recurrent pharyngitis and ocular involvement. The father of one of our patients had three recurrent episodes of facial palsy. We did not find a positive family history of MS in our sample.

Finally, we performed a small-scale molecular epidemiology study to assess the TNFRSF1A R92Q mutation as a genetic risk factor for developing MS. We found a marginally non-significant increase in the frequency of mutants carriers in the cohort of patients $(5.5 \%)$ as compared to the control group $(1.3 \%), P=0.1 ; \mathrm{OR}=4.5$; 95\% CI 0.53-40.3.

\section{Discussion}

We identified a group of five patients with MS that presented a history of recurrent autoinflammatory symptoms before the onset of their neurological disorder. All of them were heterozygous carriers of the TNFRSF1A R92Q mutation, which is the more frequent genetic defect described in TRAPS patients [4]. These are the first cases identified and reported in Argentina except for the 26 patients previously reported in Germany $[6,11]$. Although the autoinflammatory symptoms were similar in our group of patients as compared to those from the German group, we found differences in terms of treatment tolerability. Kümpfel et al. found that most of their patients experienced severe side effects during immunomodulatory treatment for MS. We did not observe this problem with the pharmacological therapy. In R92Q carriers, MS onset was more frequently detected in the brainstem. Two patients presented facial nerve palsy and the father of one proband reported recurrent episodes of facial nerve palsy. Facial nerve palsy is an uncommon MS onset symptom. Two small case-series found this symptom in about $5 \%$ of their MS patients $[12,13]$. This symptom seems to be more frequent in MS R92Q carriers. When we combined our patients with those described by Kümpfel et al., we found that about $15 \%$ of MS R92Q carriers had facial nerve palsy as MS onset symptom. However, further studies should be performed to confirm this observation. Moreover, the role of TNF $\alpha$ in idiopathic facial nerve palsy remains unclear. Other authors have measured TNF $\alpha$ levels in patients with facial nerve palsy and obtained opposite results $[14,15]$.

The pathogenicity of the R92Q mutation is a subject of debate. Its frequency in healthy cohorts has been reported in up to $2.25 \%$ of subjects [16]. Although soluble TNFRSF1A serum levels in vivo do not seem to increase with attacks, monocytes from patients bearing the R92Q substitution show TNFRSF1A membrane staining and receptor shedding comparable to those in controls [17], thus suggesting that additional pathogenic mechanisms may be operative in TRAPS. Furthermore, Lobito et al. reported that the R92Q variant TNFRSF1A behaves like wild-type TNFRSF1A, with apparently normal folding, identical surface expression and TNF binding [18]. Nevertheless, its increased frequency among patients with periodic fever suggests that it is a low-penetrance mutation rather than a benign polymorphism [4]. The R92Q allele may cause milder disease, and may often be associated with an oligosymptomatic and atypical course, including cardiac diseases [19, 20], shorter febrile bouts and rhinopharingeal involvement [21]. Our patients presented autoinflammatory symptoms atypical for classical TRAPS, such as recurrent pharyngitis which are more characteristics of other autoinflammatory syndromes like the periodic fever, aphthosis, pharyngitis, and adenitis syndrome (PFAPA). Pelagatti et al., recently reported that subjects 
carrying the R92Q mutation in comparison with those carrying TNFRSF1A structural mutations more frequently expressed a phenotype more consistent with PFAPA than TRAPS [21]. A finding that is consistent with our observation.

TNFRSF1A R92Q mutation could be considered a risk factor for developing MS. Although we and other authors in small and underpowered studies found a non-significant difference in the frequency of carriers of this mutation between patients and controls [22], the direction of the differences found and the results of a meta-analysis of genome wide association studies performed that showed this mutation as a genetic risk factor for the pathology development in more than 9000 patients support our findings [5].

\section{Conclusion}

In conclusion, we identified TNFRSF1A R92Q mutation carriers that showed the coexistence between MS and autoinflammatory symptoms. We also observed an increase in the frequency of carriers of this mutation in our population of MS patients. Our findings suggest a role of TNF $\alpha$ signaling in MS.

Acknowledgments This work was supported by grants (to MAK) from CONICET. MAK is a member of CONICET Scientific and Technological Researcher's Career. The authors thank the individuals with MS and their families for making this study possible.

\section{References}

1. Hull KM, Drewe E, Aksentijevich I, Singh HK, Wong K, McDermott EM, Dean J, Powell RJ, Kastner DL (2002) The TNF receptor-associated periodic syndrome (TRAPS): emerging concepts of an autoinflammatory disorder. Medicine (Baltimore) 81(5):349-368

2. Williamson LM, Hull D, Mehta R, Reeves WG, Robinson BH, Toghill PJ (1982) Familial Hibernian fever. Q J Med 51(204):469-480

3. Touitou I, Lesage S, McDermott M, Cuisset L, Hoffman H, Dode C, Shoham N, Aganna E, Hugot JP, Wise C, Waterham H, Pugnere D, Demaille J, de Sarrauste Menthiere C (2004) Infevers: an evolving mutation database for auto-inflammatory syndromes. Hum Mutat 24(3):194-198. doi:10.1002/humu.20080

4. Ravet N, Rouaghe S, Dode C, Bienvenu J, Stirnemann J, Levy P, Delpech M, Grateau G (2006) Clinical significance of P46L and R92Q substitutions in the tumour necrosis factor superfamily 1A gene. Ann Rheum Dis 65(9):1158-1162. doi:10.1136/ard.2005. 048611

5. De Jager PL, Jia X, Wang J, de Bakker PI, Ottoboni L, Aggarwal NT, Piccio L, Raychaudhuri S, Tran D, Aubin C, Briskin R, Romano S, Baranzini SE, McCauley JL, Pericak-Vance MA, Haines JL, Gibson RA, Naeglin Y, Uitdehaag B, Matthews PM, Kappos L, Polman C, McArdle WL, Strachan DP, Evans D, Cross AH, Daly MJ, Compston A, Sawcer SJ, Weiner HL, Hauser SL, Hafler DA, Oksenberg JR (2009) Meta-analysis of genome scans and replication identify CD6, IRF8 and TNFRSF1A as new multiple sclerosis susceptibility loci. Nat Genet 41(7):776-782. doi:10.1038/ng.401

6. Kumpfel T, Hoffmann LA, Pellkofer H, Pollmann W, Feneberg W, Hohlfeld R, Lohse P (2008) Multiple sclerosis and the TNFRSF1A R92Q mutation: clinical characteristics of 21 cases. Neurology 71(22):1812-1820. doi:10.1212/01.wnl.0000335930. 18776.47

7. Minden K, Aganna E, McDermott MF, Zink A (2004) Tumour necrosis factor receptor associated periodic syndrome (TRAPS) with central nervous system involvement. Ann Rheum Dis 63(10):1356-1357. doi:10.1136/ard.2003.016006

8. Sriram S (2008) TRAPS and MS: two diseases or an MS mimic? Neurology 70(13 Pt 2):1077-1078. doi:10.1212/01.wnl.0000307 670.62365 .08

9. TNF neutralization in MS: results of a randomized, placebocontrolled multicenter study. The Lenercept Multiple Sclerosis Study Group and The University of British Columbia MS/MRI Analysis Group (1999). Neurology 53 (3):457-465

10. McDonald WI, Compston A, Edan G, Goodkin D, Hartung HP, Lublin FD, McFarland HF, Paty DW, Polman CH, Reingold SC, Sandberg-Wollheim M, Sibley W, Thompson A, van den Noort S, Weinshenker BY, Wolinsky JS (2001) Recommended diagnostic criteria for multiple sclerosis: guidelines from the International Panel on the diagnosis of multiple sclerosis. Ann Neurol 50(1):121-127

11. Hoffmann LA, Lohse P, Konig FB, Feneberg W, Hohlfeld R, Kumpfel T (2008) TNFRSF1A R92Q mutation in association with a multiple sclerosis-like demyelinating syndrome. Neurology 70(13 Pt 2):1155-1156. doi:10.1212/01.wnl.0000296279. $98236.8 \mathrm{a}$

12. Fukazawa T, Moriwaka F, Hamada K, Hamada T, Tashiro K (1997) Facial palsy in multiple sclerosis. J Neurol 244(10): 631-633

13. Adeva-Bartolome MT, Blazquez-Estrada MT, Fermoso-Garcia J (1999) Bell's palsy as a form of presentation of multiple sclerosis. Rev Neurol 28(5):534

14. Larsson C, Bernstrom-Lundberg C, Edstrom S, Bergstrom $\mathrm{T}$ (1998) Tumor necrosis factor-alpha response and herpesvirus infection in Bell's palsy. Laryngoscope 108(8 Pt 1):1171-1176

15. Kaygusuz I, Godekmerdan A, Keles E, Karlidag T, Yalcin S, Yildiz M, Tazegul A (2004) The role of viruses in idiopathic peripheral facial palsy and cellular immune response. Am J Otolaryngol 25(6):401-406

16. D' Osualdo A, Ferlito F, Prigione I, Obici L, Meini A, Zulian F, Pontillo A, Corona F, Barcellona R, Di Duca M, Santamaria G, Traverso F, Picco P, Baldi M, Plebani A, Ravazzolo R, Ceccherini I, Martini A, Gattorno M (2006) Neutrophils from patients with TNFRSF1A mutations display resistance to tumor necrosis factor-induced apoptosis: pathogenetic and clinical implications. Arthritis Rheum 54(3):998-1008. doi:10.1002/art.21657

17. Aksentijevich I, Galon J, Soares M, Mansfield E, Hull K, Oh HH, Goldbach-Mansky R, Dean J, Athreya B, Reginato AJ, Henrickson M, Pons-Estel B, O'Shea JJ, Kastner DL (2001) The tumor-necrosis-factor receptor-associated periodic syndrome: new mutations in TNFRSF1A, ancestral origins, genotype-phenotype studies, and evidence for further genetic heterogeneity of periodic fevers. Am J Hum Genet 69(2):301-314. doi:10.1086/ 321976

18. Lobito AA, Kimberley FC, Muppidi JR, Komarow H, Jackson AJ, Hull KM, Kastner DL, Screaton GR, Siegel RM (2006) Abnormal disulfide-linked oligomerization results in ER retention and altered signaling by TNFR1 mutants in TNFR1-associated periodic fever syndrome (TRAPS). Blood 108(4):1320-1327. doi:10.1182/blood-2005-11-006783

19. Cantarini L, Lucherini OM, Cimaz R, Baldari CT, Laghi Pasini F, Galeazzi M (2010) Sacroileitis and pericarditis: atypical 
presentation of tumor necrosis factor receptor-associated periodic syndrome and response to etanercept therapy. Clin Exp Rheumatol 28(2):290-291

20. Cantarini L, Lucherini OM, Cimaz R, Baldari CT, Bellisai F, Rossi Paccani S, Laghi Pasini F, Capecchi PL, Sebastiani GD, Galeazzi M (2009) Idiopathic recurrent pericarditis refractory to colchicine treatment can reveal tumor necrosis factor receptorassociated periodic syndrome. Int $\mathrm{J}$ Immunopathol Pharmacol 22(4):1051-1058

21. Pelagatti MA, Meini A, Caorsi R, Cattalini M, Federici S, Zulian F, Calcagno G, Tommasini A, Bossi G, Sormani MP, Caroli F,
Plebani A, Ceccherini I, Martini A, Gattorno M (2011) Longterm clinical profile of children with the low-penetrance R92Q mutation of the TNFRSF1A gene. Arthritis Rheum 63(4):11411150. doi:10.1002/art.30237

22. Jenne DE, Aries PM, Einwachter S, Akkad AD, Wieczorek S, Lamprecht P, Gross WL (2007) The low-penetrance R92Q mutation of the tumour necrosis factor superfamily $1 \mathrm{~A}$ gene is neither a major risk factor for Wegener's granulomatosis nor multiple sclerosis. Ann Rheum Dis 66(9):1266-1267. doi: 10.1136/ard.2006.065987 